BMJ

Open

Gastroenterology

\title{
Does long-term use of proton pump inhibitors cause hypomagnesaemia in Japanese outpatients?
}

\author{
Yasuhito Takeda, Hisashi Doyama, Kunihiro Tsuji, Shinya Yamada, Kenichi Takemura
}

To cite: Takeda Y, Doyama $\mathrm{H}$, Tsuji K, et al. Does long-term use of proton pump inhibitors cause hypomagnesaemia in Japanese outpatients?. BMJ Open Gastro 2014;1:e000003. doi:10.1136/bmjgast-2014000003

Received 14 April 2014 Revised 10 September 2014 Accepted 19 September 2014

\section{ABSTRACT}

Aim: Hypomagnesaemia has been reported associated with long-term proton pump inhibitor (PPI) treatment. However, there have been no studies in Japanese patients. We therefore assessed the effects of long-term PPI use on serum magnesium concentrations in Japanese patients.

Methods: Data from 481 outpatients who visited our centre in October and November 2011 were reviewed to determine their underlying diseases, oral medications, including PPIs, and serum magnesium concentrations. The association between PPI use and serum Mg concentrations was assessed by multiple linear regression analysis.

Results: Serum magnesium concentrations were significantly lower in PPI users $(n=199)$ than in PPI nonusers ( $n=282 ; 1.86 \pm 0.21$ vs $1.91 \pm 0.19 \mathrm{mg} / \mathrm{dL}, p<0.01$ ). Multiple linear regression analysis showed that the presence or absence of cirrhosis and PPI use was a significant predictor of magnesium concentration. In evaluating magnesium concentrations among PPI users and non-users with and without cirrhosis, we found that mean serum magnesium concentrations in patients with cirrhosis were significantly lower in PPI users than nonusers $(1.78 \pm 0.22$ vs $1.87 \pm 0.22 \mathrm{mg} / \mathrm{dL}, \mathrm{p}=0.03)$.

Conclusions: Outpatients receiving long-term PPI treatment had significantly lower serum magnesium concentrations than those not treated with PPI. To the best of our knowledge, this study is the first to show hypomagnesaemia in Japanese patients with cirrhosis receiving long-term PPI treatment.

\section{INTRODUCTION}

Proton pump inhibitors (PPIs) are widely used to treat gastrointestinal disorders, such as gastric ulcers, duodenal ulcers and reflux oesophagitis. ${ }^{1}$ Because these drugs are well tolerated, increased numbers of patients receive long-term PPI treatment. However, PPI-associated hypomagnesaemia has been reported in several patients in the USA and Europe. $^{2}$ Magnesium is the fourth most common cation in the body. Although found primarily in the muscles and bones and a constituent of numerous body structures,

\section{Summary box}

What is already known about this subject?

- Hypomagnesaemia may result from diarrhoea or malabsorption or it can be induced by medications such as cisplatin preparations.

- The US Food and Drug Administration issued an alert stating that the long-term use of proton pump inhibitors (PPIs) may result in hypomagnesaemia.

- Statistically significant relations were observed between lower serum magnesium concentrations and PPI treatment in US inpatients.

What are the new findings?

- Statistically significant relations were observed between lower serum magnesium concentrations and PPI treatment in Japanese outpatients.

- Serum magnesium levels did not differ among the groups of patients taking the three types of PPIs.

- PPI users with cirrhosis had significantly lower serum magnesium levels than non-users with cirrhosis and than patients without cirrhosis, regardless of PPI use.

How might it impact on clinical practice in the foreseeable future?

- PPIs are frequently used in everyday medical practice. Although PPI-induced hypomagnesaemia is extremely rare, it may be life threatening in some patients.

magnesium is also essential for enzymes involved in generating ATP, thus playing a key role in regulating numerous physiological functions. ${ }^{3}$ Hypomagnesaemia may result from diarrhoea or malabsorption or it can be induced by medications such as cisplatin preparations. ${ }^{4}$ Although hypomagnesaemia may cause serious conditions, such as tetany, spasms and arrhythmias, some patients are asymptomatic. Thus, the specific incidence of hypomagnesaemia associated with PPIs remains unknown.

In May 2011, the US Food and Drug Administration issued an alert stating that the long-term use of PPIs may result in hypomagnesaemia. ${ }^{5}$ Serum magnesium concentrations were found to be significantly lower in the western inpatients and outpatients who
Correspondence to Dr Yasuhito Takeda; takeda@ipch.jp 
received long-term PPI treatment compared with those who did not. ${ }^{6} 7$ Serum magnesium concentrations of patients receiving PPIs have only been rarely investigated in Japan, and there is only one case report. ${ }^{8}$ We therefore examined the effect of long-term use of PPIs on serum magnesium concentrations in Japanese outpatients.

\section{METHODS}

We studied 1742 individuals treated as outpatients at the Ishikawa Prefectural Central Hospital between October and November 2011. Our general hospital has a focus on digestive diseases. The study participants were 481 outpatients who met the inclusion criteria as follows: age $>20$ years and no history of hospitalisation within 1 week of blood sampling. Exclusion criteria were as follows: dialysis patients and patients who were administered magnesium oxide, diuretics or cisplatin preparations that may have affected serum magnesium. Cirrhosis was defined by clinical findings, blood examination data and image diagnosis (CT or ultrasound). Outpatients underwent blood sampling in the morning after at least a $12 \mathrm{~h}$ overnight fast. We measured serum magnesium levels in units of $0.1 \mathrm{mg} / \mathrm{dL}$. Patient records were retrospectively reviewed to determine their underlying diseases (hypertension, diabetes, cirrhosis and dyslipidaemia), possibility of ingestion, oral medications used, longterm use of PPIs and serum magnesium concentrations. Patients administered a PPI for $>1$ year were regarded as PPI users, whereas those administered a PPI for $<1$ year or not at all were considered PPI non-users. At the time this study was conducted, only three PPIs were approved for clinical use in Japan: omeprazole, lansoprazole and rabeprazole. The dose of each drug was omeprazole $20 \mathrm{mg}$, lansoprazole 15 or $30 \mathrm{mg}$ and rabeprazole $10 \mathrm{mg}$. Further, during this study, none of the patients were hospitalised for hypomagnesaemia. The protocol and consent form for this study were approved by the Institutional Review Board of Ishikawa Prefectural Central Hospital, and all patients provided written informed consent.

\section{Statistical analysis}

Mean, SD and percentage with frequency were used to report continuous and discrete variables. $\chi^{2}$ test or twosample $\mathrm{t}$ test was used to assess significant differences between two groups. Multiple linear regression analysis was used to determine whether serum $\mathrm{Mg}$ concentrations were significantly associated with PPI use; the mode was adjusted for potential confounders, including age, sex, diagnosis of cirrhosis, diabetes, hypertension, dyslipidaemia and PPI. A $p$ value $<0.05$ was considered statistically significant. All statistical analyses were performed using SPSS II statistical software (SPSS Japan Inc, Tokyo, Japan).

\section{RESULTS}

Patient characteristics are shown in table 1. Serum magnesium concentrations were significantly lower in PPI users than in PPI non-users $(1.86 \pm 0.21$ vs $1.91 \pm 0.19 \mathrm{mg} / \mathrm{dL}$, $\mathrm{p}<0.01)$. The distribution of serum magnesium concentrations in PPI users and non-users is shown in figure 1. Of the 481 patients, 199 were PPI users and 282 were PPI non-users (administered a PPI or not at all: 263 patients; period of 6 months from 1 month: 15 patients; period of 1 year from 6 months: 4 patients). Their mean ages were $67.5 \pm 11.7$ and $62.8 \pm 14.6$ years, respectively $(p<0.01)$. Serum magnesium stratified by age is shown in table 2 . There were significant differences in serum magnesium in PPI users $(1.86 \pm 0.04 \mathrm{mg} / \mathrm{dL})$ and non-users (1.91 $\pm 0.04 \mathrm{mg} / \mathrm{dL}$ ) in patients aged $>61$ years.

Their underlying diseases included hypertension in $30.2 \%$ and $20.2 \%$, respectively $(\mathrm{p}<0.01)$, and dyslipidaemia in $17.6 \%$ and $7.8 \%$, respectively $(\mathrm{p}<0.01)$. There were no significant differences between PPI users and non-users in the occurrence of cirrhosis or diabetes. Serum creatinine concentrations were significantly higher in PPI users than in PPI non-users $(0.79 \pm 0.30$ vs $0.73 \pm 0.26 \mathrm{mg} / \mathrm{dL}, \mathrm{p}<0.01)$. Serum albumin concentrations did not differ significantly between PPI users and PPI non-users (table 1). Serum magnesium concentrations were similar in patients taking the PPI omeprazole $(1.86 \pm 0.24 \mathrm{mg} / \mathrm{dL}), \quad$ lansoprazole $(1.86 \pm 0.17 \mathrm{mg} / \mathrm{dL})$ and rabeprazole $(1.86 \pm 0.20 \mathrm{mg} / \mathrm{dL}$; table 3$)$.

Table 1 Patient demographic and clinical characteristics

\begin{tabular}{|c|c|c|c|c|}
\hline & & PPI users $(n=199)$ & PPI non-users $(n=282)$ & p Value \\
\hline Age & Year, mean $\pm S D$ (range) & $67.5 \pm 11.7(31-91)$ & $62.8 \pm 14.6(20-87)$ & $<0.01^{*}$ \\
\hline Sex & Male/female & $108(54.2 \%) / 91(45.8 \%)$ & $151(53.5 \%) / 131(46.5 \%)$ & NSt \\
\hline Cirrhosis & Present/absent & $55(27.6 \%) / 144(72.4 \%)$ & $57(20.2 \%) / 225(79.8 \%)$ & $0.06 \dagger$ \\
\hline Diabetes & Present/absent & $26(13.1 \%) / 173(86.9 \%)$ & $34(12.1 \%) / 248(87.9 \%)$ & NSt \\
\hline Hypertension & Present/absent & $60(30.2 \%) / 139$ (69.8\%) & $57(20.2 \%) / 225$ (79.8\%) & $<0.01 \dagger$ \\
\hline Dyslipidaemia & Present/absent & 35 (17.6\%)/164 (82.4\%) & $22(7.8 \%) / 260(92.2 \%)$ & $<0.01 \dagger$ \\
\hline Serum $\mathrm{Cr}$ & mg/dL, mean \pm SD (range) & $0.79 \pm 0.30(0.35-3.68)$ & $0.73 \pm 0.26(0.26-2.73)$ & $<0.01^{*}$ \\
\hline Serum Mg & $\mathrm{mg} / \mathrm{dL}$, mean $\pm \mathrm{SD}$ (range) & $1.86 \pm 0.21(1.2-2.5)$ & $1.91 \pm 0.19(1.1-2.5)$ & $<0.01^{*}$ \\
\hline Serum Alb & g/dL, mean $\pm S D$ (range) & $4.06 \pm 0.54(2.5-5.1)$ & $4.08 \pm 0.53(2.4-5.2)$ & $\mathrm{NS}^{\star}$ \\
\hline
\end{tabular}




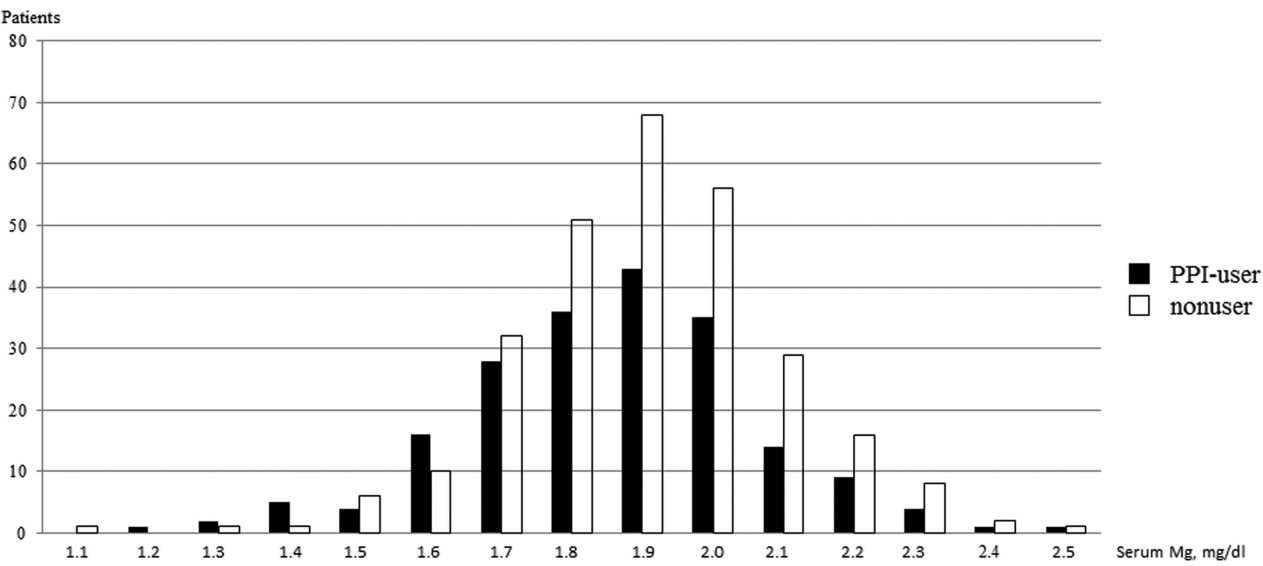

Figure 1 Distribution of serum magnesium concentrations in proton pump inhibitor (PPI) users and non-users.

\begin{tabular}{|c|c|c|c|}
\hline Age & & $\begin{array}{l}\text { Serum Mg, mg/dL, } \\
\text { mean } \pm S D\end{array}$ & p Value \\
\hline \multirow[t]{2}{*}{$20-60$} & PPI-user $(n=43)$ & $1.87 \pm 0.04$ & 0.44 \\
\hline & Non-user $(n=91)$ & $1.90 \pm 0.04$ & \\
\hline \multirow[t]{2}{*}{$61 \sim$} & PPI-user $(n=156)$ & $1.86 \pm 0.04$ & $<0.01$ \\
\hline & Non-user $(n=192)$ & $1.91 \pm 0.04$ & \\
\hline
\end{tabular}

Linear regression analysis for each patient characteristic, with use or non-use of a PPI as an explanatory variable and serum magnesium concentration as a response variable, showed that the latter differed significantly in patients with and without liver cirrhosis and with and without PPI use (table 4). Multiple linear regression analysis showed that both of these factors were significantly predictive of magnesium concentration (table 5). Serum magnesium concentration was significantly lower in patients with and without cirrhosis $(1.82 \pm 0.22$ vs 1.91 $\pm 0.19 \mathrm{mg} / \mathrm{dL}, \mathrm{p}<0.01$; figure 2 ). Among patients with cirrhosis, long-term PPI users had significantly lower serum magnesium level than PPI non-users $(1.78 \pm 0.22$ vs 1.87 $\pm 0.22 \mathrm{mg} / \mathrm{dL}, \mathrm{p}=0.03)$. Although among the noncirrhosis patients, long-term PPI users did not have significantly lower serum magnesium levels than PPI non-users $(1.89 \pm 0.20$ vs $1.92 \pm 0.18 \mathrm{mg} / \mathrm{dL})$.

\section{DISCUSSION}

We have shown here that serum magnesium concentrations were lower in Japanese outpatients who did receive long-term PPI treatment than in those who did not. Although several cases of PPI-associated hypomagnesaemia have been reported in the USA and Europe, ${ }^{2}$ 9-12 only one has been reported in Japan. ${ }^{8}$

Hypomagnesaemia has been observed in $>20 \%$ of hospitalised patients and in $65 \%$ of patients in intensive care units. $^{13-15}$ Among US inpatients, those who received long-term PPI treatment had significantly lower serum magnesium levels than PPI non-users. ${ }^{6}$ Our results in Japanese outpatients were similar to those reported for US inpatients and indicate that PPI-induced hypomagnesaemia is also highly probable among outpatients.

Magnesium is the fourth most common cation in the body, with a standard concentration range of 1.9$3.1 \mathrm{mg} / \mathrm{dL}$. Maintaining serum magnesium levels depends primarily on food intake and effective function by the kidneys and small intestine. ${ }^{3}$ Hypomagnesaemia may be caused by diarrhoea; malabsorption syndrome; malabsorption caused by excessive alcohol consumption; and nephrotoxic agents such as cisplatin, amphotericin $\mathrm{B}$ and cyclosporine. ${ }^{4}$ Because magnesium is essential for the enzymatic reactions involved in generating ATP as well as for enzymes that are involved in nucleic acid metabolism, magnesium insufficiency can affect ATP generation and neural transmission.

The mechanism of PPI-induced hypomagnesaemia has not yet been determined. Renal magnesium wasting has not been observed to date in patients with PPI-induced hypomagnesaemia, suggesting that the latter is associated with the gastrointestinal system. Magnesium is absorbed in the small intestine through active and passive transport systems. Active transcellular process mediated by the transport proteins TRPM6 and TRPM7 are normally responsible for approximately $30 \%$ of the absorption, while a passive paracellular pathway through enterocyte tight junctions is responsible for the remaining $70 \% .^{16} 17$ The functions of these TRPM6 and TRPM7 channels

Table 3 Serum magnesium concentrations in patients receiving three types of PPIs

\begin{tabular}{llll}
\hline & OPZ $(\mathbf{n}=\mathbf{7 7})$ & LPZ $(\mathbf{n}=\mathbf{3 9})$ & RPZ $(\mathbf{n = 8 3})$ \\
\hline Serum Mg mg/dL, mean \pm SD (range) & $1.86 \pm 0.24(1.3-2.5)$ & $1.86 \pm 0.17(1.5-2.3)$ & $1.86 \pm 0.20(1.2-2.5)$ \\
\hline LPZ, lansoprazole; OPZ, omeprazole; PPI, proton pump inhibitor; RPZ, rabeprazole. & &
\end{tabular}


Table 4 Univariate analysis of the association between patient characteristics and serum magnesium concentrations

\begin{tabular}{lcllr}
\hline Variables & $\begin{array}{l}\text { Standardised } \\
\text { coefficients }\end{array}$ & $\mathbf{p ~ V a l u e}$ & $\mathbf{9 5 \%} \mathbf{C l}$ & \\
\hline Age & 0.04 & 0.38 & -0.00 & 0.01 \\
Sex & 0.03 & 0.52 & -0.02 & 0.04 \\
Cirrhosis & -0.18 & 0.00 & -0.12 & -0.04 \\
Diabetes & -0.02 & 0.71 & -0.06 & 0.04 \\
Hypertension & 0.00 & 0.92 & -0.04 & 0.04 \\
Dyslipidaemia & -0.02 & 0.58 & -0.07 & 0.04 \\
PPI & -0.12 & 0.01 & -0.09 & -0.01 \\
Serum Cr & 0.03 & 0.49 & -0.03 & 0.07 \\
Serum Alb & 0.02 & 0.96 & -0.03 & 0.03 \\
\hline PPI, proton pump inhibitor. & & &
\end{tabular}

Table 5 Multiple linear regression analysis of factors predictive of lower serum magnesium concentration

\begin{tabular}{lllll}
\hline Variables & $\begin{array}{l}\text { Standardised } \\
\text { coefficients }\end{array}$ & $\mathbf{p ~ V a l u e}$ & $\mathbf{9 5 \%} \mathbf{C l}$ & \\
\hline Cirrhosis & -0.17 & 0.01 & -0.12 & -0.04 \\
PPI & -0.11 & 0.02 & -0.08 & -0.01 \\
\hline PPI, proton pump
\end{tabular}

$\mathrm{PPI}$, proton pump inhibitor.

may be impaired by a lack of protons or affected patients may be heterozygous for mutations in the TRPM6 genes. ${ }^{4}{ }^{18}$ Alternatively, the passive paracellular transport mechanism may be much less efficient.

Clinical symptoms have often been observed in patients with serum magnesium concentrations $<1.2 \mathrm{mg} / \mathrm{dL}{ }^{16}$ Of our patients, one PPI user and one PPI non-user had serum magnesium concentrations below $1.2 \mathrm{mg} / \mathrm{dL}$, although neither was clinically symptomatic. Although serum magnesium levels were significantly lower in PPI users than in non-users, few had concentrations low enough to trigger clinical symptoms. Patients with
PPI-associated hypomagnesaemia had extremely low magnesium levels. ${ }^{3} 671119$ Although additional patients should be examined, patients with extremely low magnesium levels may have factors that could further exacerbate PPI-induced hypomagnesaemia.

Serum magnesium concentrations decreased with increasing age in PPI users (table 2). Age was not a significant factor in our multivariate analysis (table 5). Therefore, hypomagnesaemia should be carefully monitored even in patients receiving long-term PPI treatment.

Serum magnesium levels did not differ among the groups of patients taking the three types of PPIs. Long-term administration of all these preparations, omeprazole, lansoprazole and rabeprazole, has been reported to result in hypomagnesaemia. Therefore, regardless of the preparation used, hypomagnesaemia should be carefully monitored in patients receiving longterm PPI treatment.

Our study also showed that cirrhosis was associated with hypomagnesaemia. Because biosynthesis reactions and reactions involving trace elements occur in the liver, patients with cirrhosis have reduced trace element concentrations as well as low serum magnesium. ${ }^{20}$ We found that serum magnesium levels were lower in patients with cirrhosis than in those without. Further, PPI users with cirrhosis had significantly lower serum magnesium levels than non-users with cirrhosis and than patients without cirrhosis, regardless of PPI use (figure 2). Although the effect of long-term PPI administration on serum magnesium levels in patients with cirrhosis has not yet been examined in detail and PPI-induced hypomagnesaemia is rare, it may be life threatening in Japanese outpatients with cirrhosis.

This study had several limitations. First, it was performed at a single institution. Since PPI-associated hypomagnesaemia is extremely rare, randomised comparative trials of serum magnesium concentrations and PPI use were virtually impossible. Second, the study included only outpatients who underwent blood sampling. Blood
Figure 2 Serum magnesium levels in cirrhosis and non-cirrhosis (PPI, proton pump inhibitor) users.

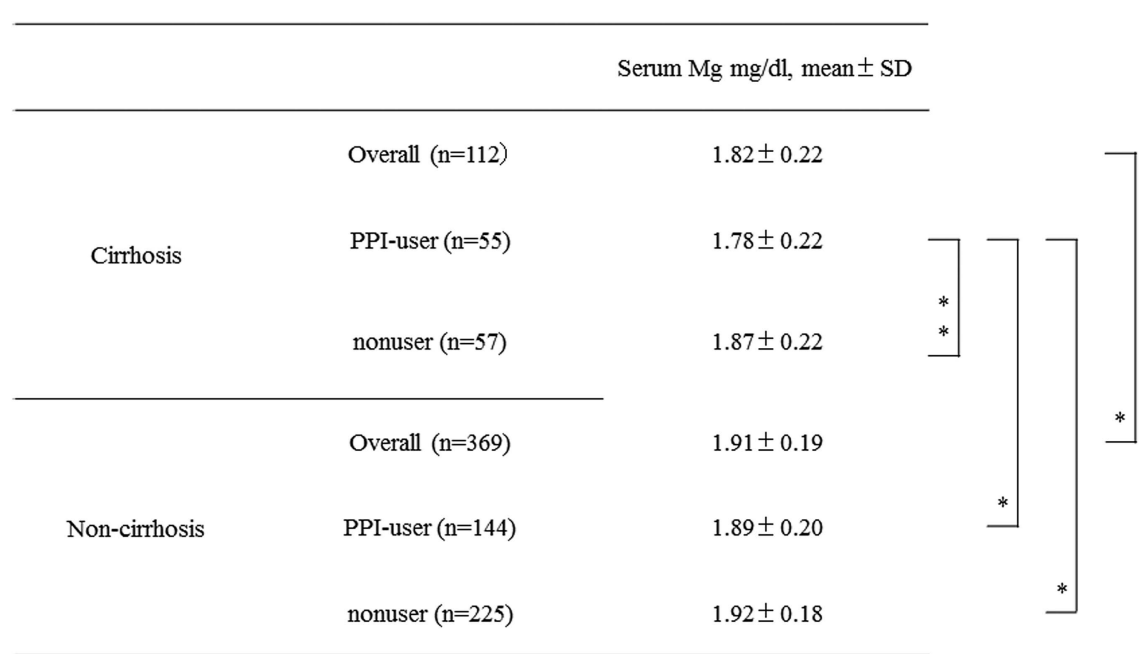

Pvalues were calculated using the t-test $\quad * \mathrm{P}=0.03, * * \mathrm{P}<0.01$ 
is sampled from only some outpatients, introducing a selection bias and preventing these results to be extrapolated to normal healthy adults. Third, a history of whether these patients took supplements containing magnesium was not evaluated completely. Although the study protocol excluded patients with a history of administration of medications that are believed to affect serum magnesium levels, supplements containing magnesium were not assessed and may have affected our results. Fourth, nutritional status (diet, alcohol intake and diarrhoea) that could affect serum magnesium levels was unknown in this study.

\section{CONCLUSION}

PPIs are frequently used in everyday medical practice. PPI-induced hypomagnesaemia may be life threatening in some patients. Some patients with PPI-induced hypomagnesaemia are asymptomatic, and this may be overlooked because of lack of information. Thus, we recommend that serum magnesium concentrations be measured periodically in outpatients receiving long-term PPI treatment. To the best of our knowledge, this study is the first to show an association between long-term PPI treatment and hypomagnesaemia in patients with cirrhosis. It is necessary to recognise the possibility of hypomagnesaemia when treating cirrhosis patients with PPIs.

Contributors YT had the original idea. KuT and SY contributed to the data analyses. KeT implemented the database. HD performed the laboratory analyses. YT wrote the initial draft and the final version of the manuscript. All the authors revised and approved the final version.

Competing interests None.

Patient consent Obtained.

Ethics approval The protocol and consent form for this study were approved by the Institutional Review Board of the Ishikawa Prefectural Central Hospital.

Provenance and peer review Not commissioned; externally peer reviewed.

Data sharing statement No additional data are available.

Open Access This is an Open Access article distributed in accordance with the Creative Commons Attribution Non Commercial (CC BY-NC 4.0) license, which permits others to distribute, remix, adapt, build upon this work non-commercially, and license their derivative works on different terms, provided the original work is properly cited and the use is non-commercial. See: http://creativecommons.org/licenses/by-nc/4.0/

\section{REFERENCES}

1. Forgacs I, Loganayagan A. Overprescribing proton pump inhibitors. BMJ 2008;336:2-3.

2. Epstein M, McGrath S, Law F. Proton pump inhibitors and hypomagnesemic hypoparathyroidism. $N$ Engl J Med 2006;355:1834-6.

3. Noronha JL, Matuschak GM. Magnesium in critical illness: metabolism, assessment and treatment. Intensive Care Med 2002;28:667-79.

4. Kuipers MT, Thang HD, Arntzenius AB. Hypomagnesaemia due to use of proton pump inhibitors - a review. Neth $J$ Med 2009;67:169-72.

5. Rockville MD. Proton pump inhibitor drugs (PPIs): Drug Safety Communication-low magnesium levels can be associated with long-term use. US Food and Drug Administration, 2011.

6. Gau JT, Yang YX, Chen R, et al. Uses of proton pump inhibitors and hypomagnesemia. Pharmacoepidemiol Drug Saf 2012;21:553-9.

7. Markovits $\mathrm{N}$, Loebstein $\mathrm{R}$, Halkin $\mathrm{H}$. The association of proton pump inhibitors and hypomagnesemia in the community setting. J Clin Pharmacol 2014:54:889-95.

8. Matsuyama J, Tsuji K, Doyama H. Hypomagnesemia associated with a proton pump inhibitor. Intern Med 2012;51:2231-4.

9. Cundy T, Dissanayake A. Severe hypomagnesaemia in long-term users of proton-pump inhibitors. Clin Endocrinol (Oxf) 2008;69:338-41.

10. Shabajee N, Lamb EJ, Sturgess I, et al. Omeprazole and refractory hypomagnesaemia. BMJ 2008;337:a425.

11. Hoorn EJ, van der Hoek J, de Man RA, et al. A case series of proton pump inhibitor-induced hypomagnesemia. Am J Kidney Dis 2010;56:112-16.

12. Mackay JD, Bladon PT. Hypomagnesaemia due to proton-pump inhibitor therapy: a clinical case series. QJM 2010;103:387-95.

13. Noronha JL, Matuschak GM. Magnesium in critical illness. Intensive Care Med 2002;28:667-79.

14. Tong GM, Rude RK. Magnesium deficiency in critical illness. J Intensive Care Med 2005;20:3-17.

15. Weisinger JR, Bellorin-Font E. Magnesium and phosphorus. Lancet 1998;352:391-6.

16. Cundy T, Mackay J. Proton pump inhibitors and severe hypomagnesaemia. Curr Opin Gastroenterol 2011;27:180-5.

17. Quamme GA. Recent developments in intestinal magnesium absorption. Curr Opin Gastroenterol 2008;24:230-5.

18. Li M, Jiang J, Yue L. Functional characterization of homo- and heteromeric channel kinases TRPM6 and TRPM7. J Gen Physiol 2006;127:525-37.

19. Hmu C, Moulik P. Severe hypomagnesaemia due to lansoprazole. BMJ Case Rep Published online: 17 Dec 2009 doi:10.1136/ bcr.07.2009.2098

20. Suzuki T, Oyama R, Hayashi E, et al. Liver disease and essential trace elements. Nihon Rinsho 1996;54:85-92. 